\title{
ALIMENTAÇÃO DA RAIA-VIOLA Zapteryx brevirostris NA BAÍA DE UBATUBA-ENSEADA, SÃO FRANCISCO DO SUL, SANTA CATARINA, BRASIL.
}

\section{Feeding habits of Zapteryx brevirostris at Ubatuba-Enseada Bay, Sa o Francisco do Sul, Santa Catarina, Brazil.}

\author{
Hugo Bornatowski ${ }^{1}$ \\ Vinícius Abilhoa ${ }^{2}$ \\ Matheus Oliveira Freitas 3
}

\section{Resumo}

Foi estudada a alimentação de Zapteryx brevirostris na baía de Ubatuba-Enseada, em Santa Catarina. Por meio de redes de arrasto com porta, 21 exemplares foram coletados em amostragens mensais entre outubro de 2003 e setembro de 2004. Uma dieta bastante diversificada foi identificada, com grande freqüência de ocorrência de Poliquetas (71,43\%) e Crustácea Decápoda (66,66\%). De acordo com o Índice de Importância Alimentar (IAi), a espécie mostrou grande preferência por Crustácea Decápoda $(47,11 \%)$ e Poliquetas $(41,10 \%)$. Os resultados encontrados concordam com outros estudos realizados na região costeira sudeste do Brasil.

Palavras-chave: Dieta; Estuário; Elasmobranchii; Rhinobatidae.

\section{Abstract}

The feeding of Zapteryx brevirostris at baía de Ubatuba-Enseada in Santa Catarina was studied. A trawl net was used for the surveys, and 21 fish were monthly collected during October (2003) and September (2004). A diversified diet was observed, and Polychaeta (71,43\%) and Crustaceans Decapods (66,66\%) were more frequents. According to the alimentary index, the diet was composed mainly by Crustaceans Decapods $(47,11 \%)$ and Polychaeta (41,10\%). Such result was similar to other studies from southern coast of Brazil.

Keywords: Diet; Estuary; Elasmobranchii; Rhinobatidae.

\footnotetext{
Hugo Bornatowski. FACIBEM - Curso de Biologia - e-mail: anequim.bio@gmail.com.

2 GPIc - Grupo de Pesquisas em Ictiofauna. Museu de História Natural Capão da Imbuia, Prefeitura de Curitiba.

3 Instituto VIDAMAR - www.vidamar.org.br.
} 


\section{Introdução}

Informações sobre a ecologia trófica de elasmobrânquios são fundamentais para o entendimento das interações biológicas de determinado ecossistema $(1,2,3)$ e diversos trabalhos têm apontado a importância de estudos com essa abordagem, tanto na avaliação de estoques pesqueiros quanto na análise holística de ecossistemas marinhos $(4,5)$.

Apesar de sua ampla distribuição na costa atlântica da América do Sul (6), a raia-viola Zapteryx brevirostris foi pouco estudada, havendo registros sobre sua estrutura populacional e aspectos reprodutivos $(7,8,9,10,11)$, e sobre sua alimentação $(12,13)$.

O objetivo deste trabalho foi trazer informações sobre a alimentação de Zapteryx brevirostris no litoral sul do Brasil, obtendo informações que contribuíssem para uma melhor compreensão sobre a interação da fauna de peixes com 0 ambiente costeiro.

\section{Material e Métodos}

Foram realizadas amostragens mensais entre de outubro de 2003 a setembro de 2004, sempre na lua de sizígia (cheia), na baía de UbatubaEnseada, no município de São Francisco do Sul -

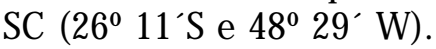

Os exemplares foram coletados por meio de nove arrastos mensais consecutivos, com duração de 5min cada, realizados por embarcação artesanal denominada de "arrasteiro", que apresenta $8 \mathrm{~m}$ de comprimento e redes de arrasto com portas com $7 \mathrm{~m}$ de comprimento e $3 \mathrm{~m}$ de altura, com malha de $3 \mathrm{~cm}$ entre nós consecutivos na região do ensacador.

Após a coleta, os indivíduos foram fixados em formol $10 \%$ e, posteriormente, conservados em álcool 70\%. Em laboratório, eles foram mensurados para obtenção do comprimento padrão, pesados e dissecados para a retirada do aparelho digestório. Os tratos estomacais foram removidos e fixados em formol $4 \%$, sendo posteriormente conservados em álcool 70\%.

No laboratório de Ictiologia do Museu de História Natural Capão da Imbuia, os conteúdos estomacais foram analisados sob microscópio estereoscópico e a identificação dos itens foi reali- zada com auxílio de bibliografia específica e consulta a especialistas.

Para a análise da importância dos itens alimentares identificados, foi utilizado o método de Freqüência de Ocorrência, que avalia percentual de estômagos em que determinado item alimentar ocorre e o método de Número de Pontos, onde a contribuição de cada item é determinada pela proporção de quadrículas ocupadas pelo item em uma superfície plana quadriculada em relação ao número total de quadrículas ocupadas pelo conteúdo $(14,15)$. Para análise da importância efetiva de cada item na alimentação da espécie foi utilizado o Índice Alimentar (IAi) proposto por Kawakami e Vazzoler (16).

\section{Resultados e Discussão}

A análise de 21 estômagos de Zapteryx brevirostris mostrou uma dieta bastante diversificada, composta por Poríferos, Crustácea Decápoda, Poliquetas, peixes e Cefalocordados (Tabela 1).

Os itens alimentares com maior freqüência de ocorrência nos estômagos foram Restos de Poliquetas (71,43\%), Crustácea Decápoda (66,66\%), Poliquetas da Ordem Phyllodocida (33,33\%) e restos de Crustácea $(38,1 \%)$ (Tabela 2). Já de acordo com o Índice de Importância Alimentar, os itens mais representativos na dieta de Z. brevirostris foram Crustácea Decápoda (47,11\%), Poliquetas $(41,10 \%)$ e restos de Crustácea $(11,25 \%)$ (Figura $1)$.

Estes resultados encontrados concordam com a tendência alimentar relatada para a espécie por Soares et al. (13), ou seja, de uma dieta baseada principalmente em crustáceos e poliquetas.

A grande ocorrência de restos de Poliquetas e Crustáceos na dieta de Zapteryx brevirostris pode refletir também a função trituradora desempenhada pelo aparato bucal. Em um estudo realizado com outro Rhinobatidae (Rhinobatos lentiginosus), foi constatado que, no momento da predação, a presa é manipulada pela boca e é expelida e recapturada diversas vezes, sendo altamente triturada pelas maxilas (17).

Muito embora a participação de poliquetas na dieta de Z. brevirostris na área de estudo tenha sido expressiva, uma supremacia de crustáceos decápodes foi registrada, o que concorda com es- 
tudos realizados na região costeira sudeste do Brasil $(6,8,13)$.

O hábito de alimentação à base de crustáceos decápodes foi encontrado em outros peixes batóideos também $(17,18)$, o que segundo Bacescu e Queiroz (19) deve-se ao fato destes grupos de hábitos demersais explorarem o mesmo ambiente.
De fato, representantes da ordem Rhinobatiformes (raias-viola) são estritamente costeiros e as quatro espécies brasileiras estão associadas às águas costeiras e estuarinas ao longo de toda a costa, representando cerca de $5 \%$ da fauna de elasmobrânquios costeiros do Brasil (20).

TABELA 1 - Iten s identificados nos conteúdos estomacais de 21 exemplares de Zapteryx brevirostris capturados na baía de Ubatuba-Enseada, São Francisco do Sul, SC, Brasil (2003/2004).

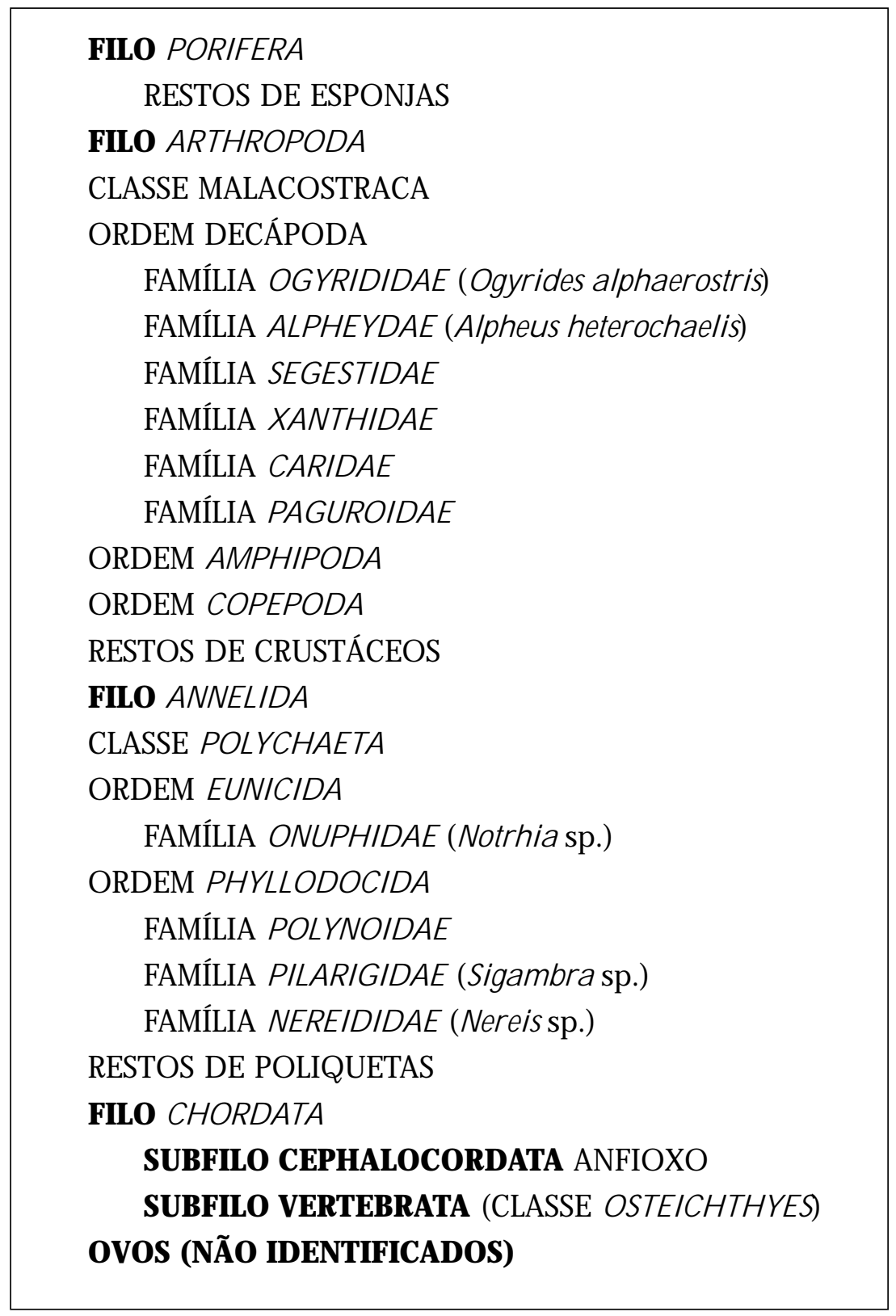


TABELA 2 - Índices de Importância Alimentar (IAi) das principais categorias de itens identifica dos nos tratos digestivos de 21 exemplares Z. brevirostris capturados na baía de Ubatuba-Enseada, São Francisco do Sul, SC, Brasil. (FO) - Freqüência de Ocorrência; (P) Método de Pontos.

\begin{tabular}{|l|l|l|l|}
\hline ITENS ALIMENTARES & FO(\%) & P(\%) & IAi(\%) \\
\hline CLASSE MALACOSTRACA & & & \\
ORDEM DECAPODA & 66,66 & 35,37 & 47,11 \\
ORDEM COPEPODA & 4,76 & 0,14 & 0,01 \\
ORDEM AMPHIPODA & 4,76 & 0,07 & 0,01 \\
RESTOS CRUSTÁCEOS & 38,10 & 14,78 & 11,25 \\
CLASSE POLIQUETA & & & \\
ORDEM EUNICIDA & 4,76 & 0,06 & 0,01 \\
ORDEM PHYLODOCIDA & 33,33 & 9,06 & 6,03 \\
RESTOS POLIQUETAS & 71,43 & 24,57 & 35,06 \\
FILO PORIFERA & 4,76 & 2,05 & 0,19 \\
CLASSE OSTEICHTHYES & 9,52 & 1,49 & 0,28 \\
SUBCLASSE CEPHALOCORDATA & 4,76 & 0,28 & 0,03 \\
OVOS & 9,52 & 0,07 & 0,01 \\
\hline
\end{tabular}

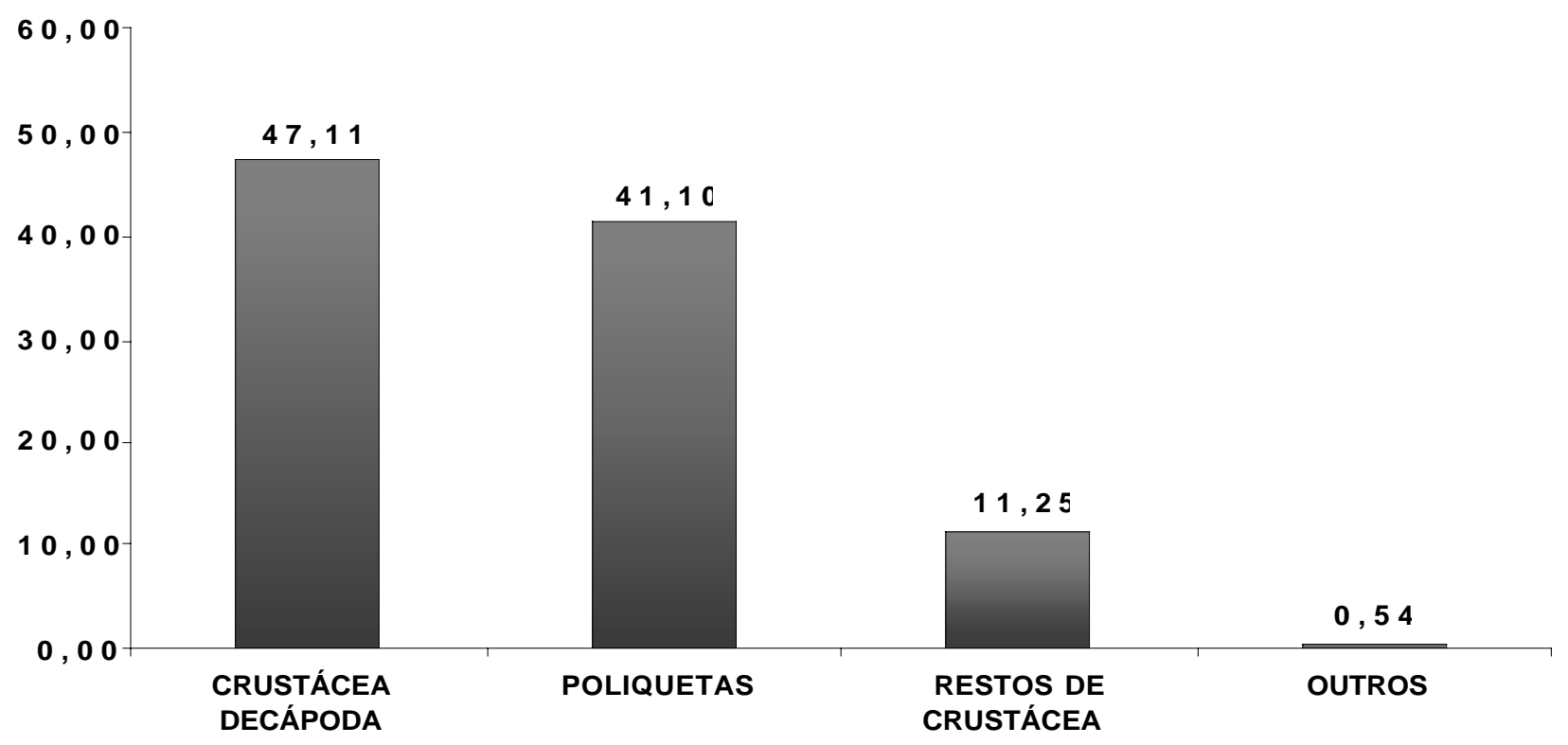

FIGURA 1 - Índice de Importância Alimentar dos itens mais representativos em 21 estômagos de Zapteryx brevirostris capturados na baía de Ubatuba-Enseada, São Francisco do Sul, SC, Brasil (2003/2004). 


\section{Conclusão}

Zapteryx brevirostris apresenta hábito exclusivamente bentônico, alimentando-se preferencialmente de crustáceos e poliquetas com destaque aos crustáceos Decápodes.

\section{Agradecimentos}

Agradecemos a Rosemary Aparecida Brogim (Laboratório de Ecologia Bêntica, Museu de História Natural Capão da Imbuia) pelo auxílio na identificação dos crustáceos e anelídeos poliquetas, e a Maunício Hostim-Silva (Univali), pelo apoio durante a realização do projeto.

\section{Referências}

1. Gulland JA. Fish stock assessment: a manual of basic methods. New York (Usa): John Wiley; 1983.

2. Pauly D. Fish population dynamics in tropical waters: a manual for use with programmable calculators. Manila: ICLARM; 1984.

3. Caddy JF, Sharp GD. An ecological framework for marine fishery investigations. FAO Fish Tech Pap 1986; 283:1-152.

4. Jones R. Ecosystems, food chains and fish yields. In: Pauly D and Murphy GI, editors. Theory and management of tropical fisheries. ICLARM Conf Proc 1982; 9:195-239.

5. Blaber SJM, Bulman CM. Diets of fishes of the upper continental slope of eastern Tasmania: content, calorific values, dietary overlap and trophic relationships. Mar Biol 1987; 95:345-356.

6. Figueiredo JL. Manual de peixes Marinhos do Sudeste do Brasil. I. Introdução. Cações, raias e quimeras. São Paulo (Brasil): Museu de Zoologia da Universidade de São Paulo; 1977.

7. Machado VO, Sant'Anna VB. Dieta da raia Zapteryx brevirostris (Müller \& Henle, 1841) (Rhinobatiformes: Rhinobatidae) no litoral de Niterói - RJ: dados preliminares. In: Resumos do XIV Encontro Brasileiro de Ictiologia. São Leopoldo (RS): Sociedade Brasileira de Ictiologia / Unisinos; 2001. p.14.
8. Pasquino AF, Gadig OBF. Estudo da alimentação da raia-viola de focinho curto Zapteryx brevirostris da costa de São Paulo (Chondrichthyes, Rhinobatidae). In: Livro de Resumos do Congresso Brasileiro de Oceanografia e XVI Semana Nacional de Oceanografia. Itajaí: Univali; 2004. p.189.

9. Cortellete GM. Estrutura populacional e abundância da raia-viola-de-focinho-curto, Zapteryx brevirostris, na plataforma continental em frente à baía de Paranaguá, Paraná [Monografia]. Curitiba (PR): Universidade Federal do Paraná; 2005.

10. Batista VS. Desenvolvimento Sexual de Zapteryx brevirostris (Müller \& Henle, 1841), no Litoral do Rio de Janeiro, Brasil. Rev Brasil Biol 1987; 47(3):301-307.

11. Batista VS. Aspectos quantitativos da fecundidade e do desenvolvimento embrionário da raia Zapteryx brevirostris Müller \& Henle 1841 (Pisces, Rhinobtidae) da Enseada de Itaipu, Niterói, Rio de Janeiro. Rev Brasil Biol 1991; 51(3):495-501.

12. Amaral ACZ, Migotto AE. Importância dos anelídeos poliquetas na alimentacao da macrofauna demersal e epibentônica da região de Ubatuba. Bolm Inst O ceanogr 1980; 29(2):3135.

13. Soares LSH, Rossi-Wongtschowski CLDB, Alvares LMC, Muto EY, Gasalla MLA. Grupos tróficos de peixes demersais da plataforma continental interna de Ubatuba, Brasil. I. Chondrichthyes. Bolm Inst Oceanogr 1992; 40(1/2):79-85.

14. Hynes HBN. The food of fish-water sticklebacks (Gasteronotus aculeatus and Pygosteus pungitius), with a review of methods used in studies of the food of fishes. J Anim Ecol 1950; 19:36-57.

15. Hyslop EJ. Stomach contents analisys - a review of methods and their application. J Fish Biol 1980; 17:411-429.

16. Kawakami E, Vazzoler G. Método gráfico e estimativa de índice alimentar aplicado no estudo de alimentação de peixes. Bol Inst Oceanog 1980; 29(2):205-207. 
17. Wilga CD, Motta PJ. Feeding mechanism of the atlantic guitarfish Rhinobatos lentiginosus: modulation of kinematic and motor activity. The Journal of Experimental Biology 1998; 201:31673184.

18. Carqueija CRG, Filho JJS, Gouvea EP, Queiroz EL Decápodos (Crustácea) utilizados na alimentação de Dasyatis guttata (Bloch e Schneider) (Elasmobranchii, Dasyatididae) na área de influência da Estação Ecológica Ilha do Medo, Baía de Todos os Santos, Bahia, Brasil. Revta Bras Zool 1995; 12(4): 833-838.
19. Bascescu M, Queiroz EL. The contribution of Cumacea in the feeding of Rajidae Sympterigia acutata and S. bonapartei from Rio Grande do Sul - S. Brazil. Trav Mus Hist Nat Grigore Antipa 1985; 27:1-8.

20. Lessa R, Santana FM, Rincón G, Gadig OBF, ElDeir ACA. Avaliação a Ações Prioritárias para a Conservação de Biodiversidade da Zona Costeira e Marinha. Biodiversidade de Elasmobrânquios do Brasil [Online] 1998. Disponível em: URL: http://www.bdt.org.br/ workshop/costa/ elasmo.

Recebido em / Received: June 6, 2005.

Revisado em / Revised: July 22, 2005. Aceito em / Accepted: September 5, 2005. 